\title{
Mesh Refinement For Stabilized Convection Diffusion Equations
}

\author{
B. Achchab ${ }^{1}$ *, M. El Fatini ${ }^{1,2}$ and A. Souissi ${ }^{3}$ \\ ${ }^{1}$ Hassan $1^{\text {st }}$ University, LM2CE, ESTB and FSJES, B.P. 218, Berrechid, Morocco \\ ${ }^{2}$ Hassan II University -Mohammadia, LAMS, L3A, FSBM, B.P. 7955, Casablanca, Morocco \\ ${ }^{3}$ Mohammed V-Agdal University, GAN, LMA, FSR and LERMA, EMI, B.P. 1014, Rabat, Morocco
}

\begin{abstract}
We derive a residual a posteriori error estimates for the subscales stabilization of convection diffusion equation. The estimator yields upper bound on the error which is global and lower bound that is local.
\end{abstract}

Key words: a posteriori error estimates, convection diffusion equation, stabilization AMS subject classification: $65 \mathrm{~N} 15,65 \mathrm{~N} 30,65 \mathrm{~N} 50$

\section{Introduction}

In a water pollution problem, early detection is most important to take swift and appropriate measures. The prediction of the contamination scale by rapid actions is also important to help prevent or mitigate related damage at downstream. The accurate method to predict the scale of the pollution impact is needed. The transport and diffusion of ingredient in a flow field is a basic process in modelling air/water pollution propagation. This process is governed by the convection-diffusion equation and subjected to random fluctuations. Numerical methods are important in determining the concentration of pollutants and understanding the change of pollutant concentration.

In many applications areas involving the mathematical modelling of convection diffusion and reaction processes, diffusion can be small (compared to the convection and the reaction coefficients), degenerate, or even identically equal to zero in subregions of the computational domain. Stabilized finite element methods are particularly interesting for those cases [3-8]. Here we consider such convection-diffusion equation stabilized by subscales method with adaptive

${ }^{*}$ Corresponding author. E-mail: achchab@yahoo.fr 
mesh refinement to solve in a domain $\Omega \subset \mathbb{R}^{n}, n=2,3$, the following concentration equation

$$
\begin{aligned}
\operatorname{div}(\beta u-\varepsilon \nabla u)+\sigma u & =f \quad \text { in } \Omega, \\
u & =0 \text { on } \partial \Omega,
\end{aligned}
$$

where $u$ is the concentration, $\varepsilon>0$ is the diffusion coefficient, $\beta \in\left(W^{1, \infty}(\Omega)\right)^{n}$ is the pore water velocity, $\sigma \in L^{\infty}(\Omega)$ is the reaction term and $f \in L^{2}(\Omega)$ is the production rate. We suppose that the pore water velocity $\beta$ is solenoidal in space. We define the operator

$$
\mathcal{L}=\operatorname{div}(\beta u-\varepsilon \nabla u)+\sigma u .
$$

The weak form of the problem is to seek $u \in H_{0}^{1}(\Omega)$, such that

$$
a(u, w)=l(w) \quad \forall w \in H_{0}^{1}(\Omega),
$$

where $a(u, w)=\varepsilon(\nabla u, \nabla w)+(\beta . \nabla u+\sigma u, w)$ and $l(w)=(f, w)$.

\section{Subscales finite element stabilization}

Let $V_{h, 0} \subset H_{0}^{1}(\Omega)$, be a conforming finite element space of piecewise polynomials. The standard Galerkin approximation of (1.4) is to find $u_{h} \in V_{h}$, such that

$$
a\left(u_{h}, w_{h}\right)=l\left(w_{h}\right) \quad \forall w_{h} \in V_{h, 0} .
$$

The standard Galerkin method lacks stability for near-hyperbolic problem. The key idea of the multiscale formulation in [7] is to consider $V_{0}=H_{0}^{1}(\Omega)$ as the direct sum of two spaces

$$
V_{0}=V_{h, 0} \oplus \widetilde{V}
$$

where $V_{h, 0}$ is the space of resolved scales and $\widetilde{V}$ is the space of subgrid scales. We can now split the problem (2.1):

$$
\begin{aligned}
a\left(u_{h}+\widetilde{u}, w_{h}\right) & =l\left(w_{h}\right) \quad \forall w_{h} \in V_{h, 0}, \\
a\left(u_{h}+\widetilde{u}, \widetilde{w}\right) & =l(\widetilde{w}) \quad \forall \widetilde{w} \in \widetilde{V} .
\end{aligned}
$$

The subscales are modeled analytically using subscales approximation in [5], $\widetilde{u}=\tau_{T} \mathcal{R} u_{h}$, where $\tau_{T}$ is called the relaxation time and $\mathcal{R} u_{h}:=\gamma-\mathcal{L} u_{h}$ is the grid scale residual. After integration by parts on each element, the equation for the grid scales reads

$$
a\left(u_{h}, w_{h}\right)+\sum_{T \in \mathcal{T}_{h}}\left(\widetilde{u}, \mathcal{L}^{*} w_{h}\right)=l\left(w_{h}\right) \quad \forall w_{h} \in V_{h, 0},
$$

where $\mathcal{L}^{*}$ is the adjoint of $\mathcal{L}$. the final equation for the resolved scales includes the usual Galerkin terms and some additional volume integrals evaluated element by element. Since the subscales $\widetilde{u}$ are proportional to the grid scale residual, the method is residual-based and therefore, automatically consistent 


\section{A posteriori error estimates}

The purpose is to get local lower and global upper bounds for the error measured in the energy norm

$$
\|v\|^{2}=\varepsilon\|\nabla v\|_{\Omega}^{2}+\|v\|_{\Omega}^{2} .
$$

Let $\mathcal{E}_{h}$ denote the set of all $(n-1)$-faces in $\mathcal{T}_{h}$. This set can be split into $\mathcal{E}_{h}=\mathcal{E}_{h, \Omega} \cup \mathcal{E}_{h, D}$, where $\mathcal{E}_{h, \Omega}$ and $\mathcal{E}_{h, D}$ refer to interior faces and Dirichlet boundary faces, respectively. For all $E \in \mathcal{E}_{h, \Omega}$ and for all $\phi$ which is piecewise smooth, $[\phi]_{E}$ denotes the jump of $\phi$ across $E$ (the sign of this quantity is irrelevant in the sequel). For all $S \in \mathcal{T}_{h} \cup \mathcal{E}_{h}$, let $\alpha_{S}=\min \left\{h_{S} \varepsilon^{-\frac{1}{2}}, 1\right\}$, where $h_{S}$ denotes the diameter of $S$. Denote by $f_{h}, \beta_{h}$ and $\sigma_{h}$ the $L^{2}$-projection of the data $f, \beta$ and $\sigma$ onto the space of piecewise constant functions on $\mathcal{T}_{h}$. Define the elementwise residual estimators as

$$
\eta_{T}^{2}=\alpha_{T}^{2}\left\|R_{T}\right\|_{T}^{2}+\sum_{E \in \mathcal{E}_{h} ; E \subset \partial T} \varepsilon^{-\frac{1}{2}} \alpha_{E}\left\|R_{E}\right\|_{E}^{2},
$$

where

$$
R_{T}=f_{h}+\varepsilon \triangle u_{h}-\beta_{h} \nabla u_{h}-\sigma_{h} u_{h},
$$

and define also the oscillation term for $T \in \mathcal{T}_{h}$ by

$$
D_{T}=\left(f-f_{h}\right)+\left(\beta-\beta_{h}\right) \nabla u_{h}+\left(\sigma-\sigma_{h}\right) u_{h} .
$$

Finally, define the elementwise data oscillation estimator as

$$
\Theta_{T}^{2}=\alpha_{T}^{2}\left\|D_{T}\right\|_{T}^{2}
$$

For $T \in \mathcal{T}_{h}$, let $\omega_{T}=\bigcup_{\emptyset \neq T \cap T^{\prime} \in \mathcal{E}_{h, \Omega}} T^{\prime}$, and set $\Theta_{\omega_{T}}^{2}=\sum_{T^{\prime} \in \omega_{T}} \Theta_{T^{\prime}}^{2}$. We proceed with the same strategy as in $[1,2,9]$ using the estimates of the Clément operator and the techniques of bubble functions we get the following results

Theorem 1. Let $u$ and $u_{h}$ be the unique solutions of (1.4) and (2.5) respectively. Then,

$$
\left\|u-u_{h}\right\|^{2} \leq \sum_{T \in \mathcal{T}_{h}}\left[\eta_{T}^{2}+\Theta_{T}^{2}\right]
$$

and for all $T \in \mathcal{T}_{h}$,

$$
\eta_{T} \leq\left(1+\varepsilon^{-\frac{1}{2}} \alpha_{T}\right)\left\|u-u_{h}\right\|_{\omega_{T}}+\Theta_{\omega_{T}}
$$

Proof. Owing to the coercivity of $a(.,$.$) , we have$

$$
\begin{aligned}
\left\|u-u_{h}\right\| & \leq \sup _{w \in H_{D}^{1}(\Omega) \backslash\{0\}} \frac{a\left(u-u_{h}, w\right)}{\|w\|} \\
& \leq \sup _{w \in H_{D}^{1}(\Omega) \backslash\{0\}} \frac{a\left(u-u_{h}, w-I_{h} w\right)}{\|w\|}+\sup _{w \in H_{D}^{1}(\Omega) \backslash\{0\}} \frac{a\left(u-u_{h}, I_{h} w\right)}{\|w\|},
\end{aligned}
$$


where $I_{h}$ is the quasi-Clément operator [9]. The first term yields

$$
a\left(u-u_{h}, w-I_{h} w\right) \leq\left\{\sum_{T \in \mathcal{T}_{h}}\left[\eta_{T}^{2}+\Theta_{T}^{2}\right]\right\}^{\frac{1}{2}}\|w\| .
$$

For the second term, we set $w_{h}:=I_{h} w$, then we get

$$
a\left(u-u_{h}, I_{h} w\right)=-\sum_{T \in \mathcal{T}_{h}}\left(\tau_{T} \mathcal{R} u_{h}, \mathcal{L}^{*} w\right)_{T} .
$$

A scaling argument, the inverse inequality and the properties of the quasi-Clément operator yield for all $w \in H_{0}^{1}(\Omega)$ that

$$
\begin{aligned}
\left\|I_{h} w\right\|_{0, T} & \leq\|w\|_{0, T} \leq\|w\|_{\omega_{T}}, \\
\left\|\nabla I_{h} w\right\|_{0, T} & \leq \kappa h_{T}^{-1} \alpha_{T}\|w\|_{0, T}, \\
\kappa\left\|\Delta I_{h} w\right\|_{0, T} & \leq \kappa h_{T}^{-2} \alpha_{T}\|w\|_{0, T},
\end{aligned}
$$

using the fact that $\tau_{T} \leq \min \left\{|\nu|_{\infty, T}^{-1} h_{T}, h_{T}^{2} \kappa^{-1}\right\}$ we get

$$
a\left(u-u_{h}, I_{h} w\right) \leq\left\{\sum_{T \in \mathcal{T}_{h}} \alpha_{T}^{2}\left\|\rho_{T}\right\|_{T}^{2}+\sum_{T \in \mathcal{T}_{h}} \Theta_{T}^{2}\right\}^{\frac{1}{2}}\|w\|,
$$

and then the upper bound (3.3) follows. Now, we bound locally the residual estimator $\eta_{T}$ in terms of the approximation error and the data oscillation estimator. For $T \in \mathcal{T}_{h}$, let

$$
\omega_{T}=\bigcup_{\emptyset \neq T \cap T^{\prime} \in \mathcal{E}_{h, \Omega}} T^{\prime},
$$

and set $\Theta_{\omega_{T}}^{2}=\sum_{T^{\prime} \in \omega_{T}} \Theta_{T^{\prime}}^{2}$. The same notation is used for the energy norm. Using the technique of bubble functions [9], we get the lower bound (3.4) as proved in [9].

\section{Numerical studies}

Under simplifying assumptions, we solve the problem (1.1) in $\Omega=[0,1]^{2} \subset \mathbb{R}^{2}$ with parameters $\beta=(1,1)^{T}, \varepsilon=10^{-4}, \sigma=1$ and $f=1$. Standard Galerkin finite element produces a globally oscillatory solution, while the solution obtained by subscales stabilization method is non-oscillatory. To capture the remaining interior layers we combine stabilization and mesh refinement. In Figure 1 , adaptive meshes and the corresponding solution (totally non-oscillatory) are presented.

\section{Acknowledgements}

This work is supported in part by Volkswagen Foundation through Grant number I/79315, Hydro 3+3 Project, the French-Moroccan Project A.I number M.A/05/115 and CNRST (Projet d'établissement, Université Hassan $1^{\text {er }}$ Settat, Ministère de l'enseignement supérieur, Maroc) 


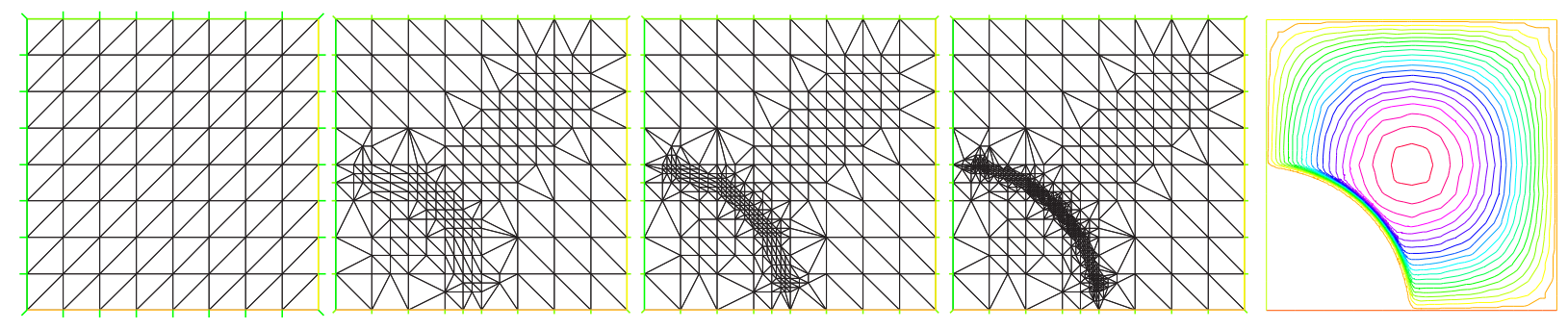

Figure 1: Successive meshes refinement and the corresponding solution totally non-oscillatory.

\section{References}

[1] B. Achchab, M. El Fatini, A.Ern, A. Souissi. Adaptive mesh for algebraic orthogonal subscale stabilization of convective dispersive transport. C. R. Math. Acad. Sci. Paris., 346 (2008), 1187-1190.

[2] B. Achchab, M. El Fatini, A.Ern, A. Souissi. A posteriori error estimator for subgrid viscosity stabilisation applied to convection-diffusion problem. AML, 22 (2009), No. 9, 1418-1424.

[3] F. Brezzi, A. Russo. Chosing bubbles for advection-diffusion problems. Math. Model. and Meth. Appl. Sci., 4 (1994), 571-587.

[4] A. N Brooks, T. J. R. Hughes. Streamline Upwind/Petrov Galerkin formulation for convection dominated flows with particular emphasis on the incompressible Navier-Stokes equations. Model. Comput. Methods Appl. Mech. Engrg., 32 (1982), 1-3.

[5] R. Codina. On stabilized finite element methods for linear systems of convection-diffusionreaction equations. Comp. Meth. Appl. Mech. Engrg., 188 (2000), 61-82.

[6] J. L. Guermond. Subgrid Stabilization of Galerkin approximations of linear monotone operators. Journal of Numerical Analysis (IMA), 21 (2001), 165-197.

[7] T. J. R. Hughes. Multiscale phenomena: Green's functions, the Dirichlet-to-Neumann formulation, subgrid-scale models, bubbles and the origin of stabilized methods. Comp. Meth. Appl. Mech. Engrg., 127 (1995), 387-401.

[8] O. Pironneau. On the transport-diffusion algorithm and its applications to the Navier-Stokes equations. Numer. Math., 38 (1982), 309-332.

[9] R. Verfürth. A posteriori error estimators for convection-diffusion equations. Numer. Math., 80 (1998), 641-663. 